\title{
Phase-locking regimes of photonic crystal nanocavity laser arrays
}

\author{
Skovgård, Troels Suhr; Kristensen, Philip Trøst; Mørk, Jesper
}

Published in:

Applied Physics Letters

Link to article, DOI:

$10.1063 / 1.3671151$

Publication date:

2011

Document Version

Publisher's PDF, also known as Version of record

Link back to DTU Orbit

Citation (APA):

Skovgård, T. S., Kristensen, P. T., \& Mørk, J. (2011). Phase-locking regimes of photonic crystal nanocavity laser arrays. Applied Physics Letters, 99(25), 251104. https://doi.org/10.1063/1.3671151

\section{General rights}

Copyright and moral rights for the publications made accessible in the public portal are retained by the authors and/or other copyright owners and it is a condition of accessing publications that users recognise and abide by the legal requirements associated with these rights.

- Users may download and print one copy of any publication from the public portal for the purpose of private study or research.

- You may not further distribute the material or use it for any profit-making activity or commercial gain

- You may freely distribute the URL identifying the publication in the public portal

If you believe that this document breaches copyright please contact us providing details, and we will remove access to the work immediately and investigate your claim. 


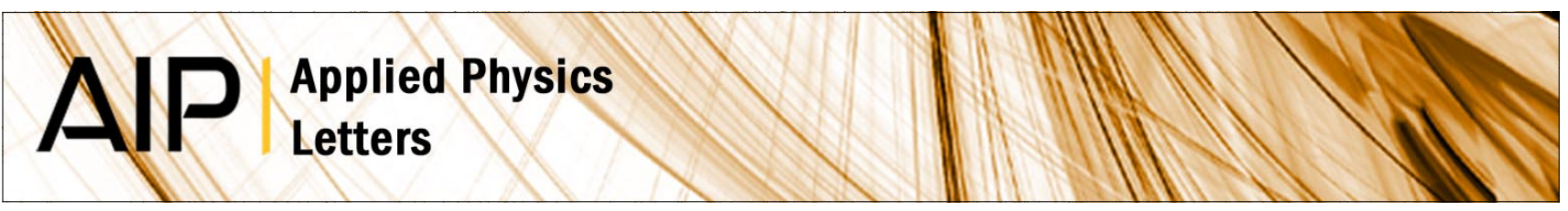

\section{Phase-locking regimes of photonic crystal nanocavity laser arrays}

T. Suhr, P. T. Kristensen, and J. Mørk

Citation: Appl. Phys. Lett. 99, 251104 (2011); doi: 10.1063/1.3671151

View online: http://dx.doi.org/10.1063/1.3671151

View Table of Contents: http://apl.aip.org/resource/1/APPLAB/v99/i25

Published by the American Institute of Physics.

\section{Related Articles}

High power, continuous wave, quantum cascade ring laser Appl. Phys. Lett. 99, 261104 (2011)

High coherent bi-chromatic laser with gigahertz splitting produced by the high diffraction orders of acousto-optic modulator used for coherent population trapping experiments

Rev. Sci. Instrum. 82, 123104 (2011)

A dual-mode quantum dot laser operating in the excited state Appl. Phys. Lett. 99, 231110 (2011)

A laser system for the parametric amplification of electromagnetic fields in a microwave cavity

Rev. Sci. Instrum. 82, 115107 (2011)

Efficient continuous wave deep ultraviolet Pr3+:LiYF4 laser at $261.3 \mathrm{~nm}$

Appl. Phys. Lett. 99, 181103 (2011)

\section{Additional information on Appl. Phys. Lett.}

Journal Homepage: http://apl.aip.org/

Journal Information: http://apl.aip.org/about/about_the_journal

Top downloads: http://apl.aip.org/features/most_downloaded

Information for Authors: http://apl.aip.org/authors

\section{ADVERTISEMENT}

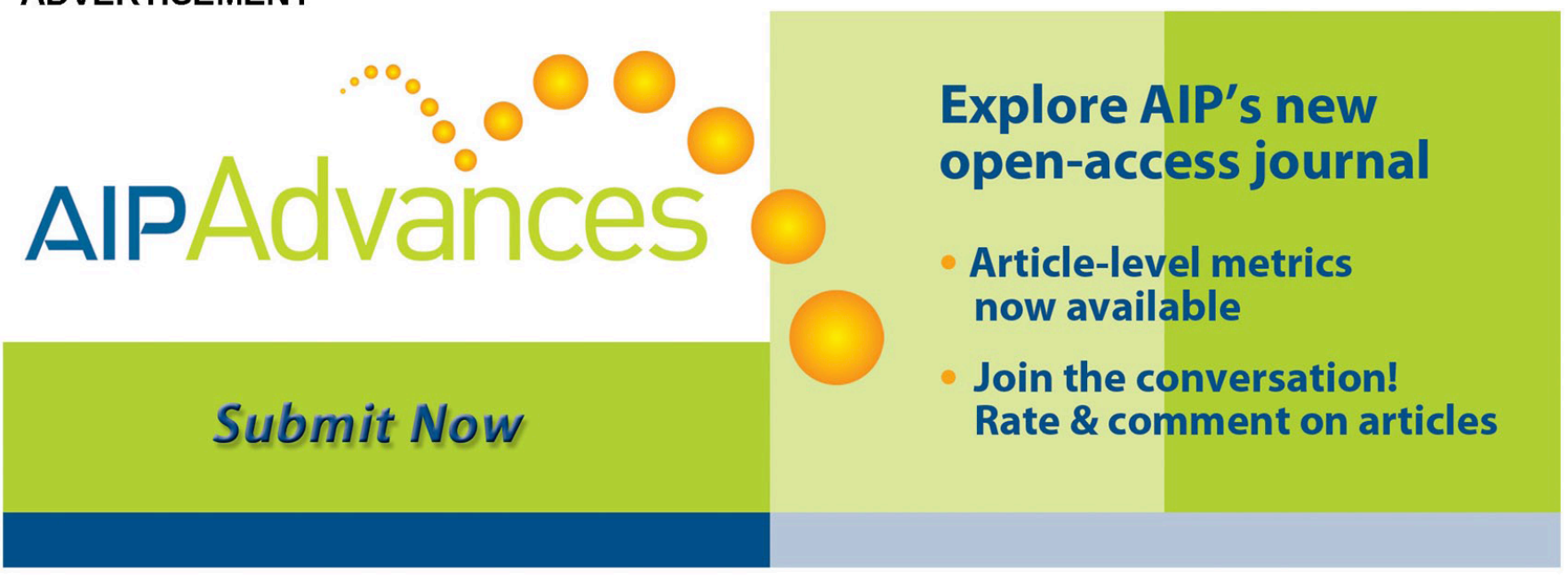




\title{
Phase-locking regimes of photonic crystal nanocavity laser arrays
}

\author{
T. Suhr, ${ }^{\text {a) }}$ P. T. Kristensen, and J. Mørk \\ DTU Fotonik, Department of Photonics Engineering, Technical University of Denmark, Ørsteds Plads 343, \\ DK2800 Kgs. Lyngby, Denmark
}

(Received 8 September 2011; accepted 29 November 2011; published online 20 December 2011)

We model and analyze the dynamical properties of coupled photonic crystal nanocavity lasers. The model includes Purcell enhancement of the spontaneous emission and intercavity coupling. The coupling strength between neighboring cavities is an essential parameter, and by performing finite-difference time-domain calculations, the typical coupling strength is extracted for realistic structures. Phase-locking regimes are identified, and their stability with respect to parameter variation is investigated. The results suggest that quantum well devices are not well suited for phase-locked nanocavity laser array devices. (c) 2011 American Institute of Physics. [doi:10.1063/1.3671151]

Photonic crystal $(\mathrm{PhC})$ lasers promise high efficiency and ultra-fast modulation speeds due to high Purcell and spontaneous emission factors $(\beta)$ but have so far been limited by their low output power. ${ }^{1-4}$ Photonic crystal laser arrays have been suggested as a way to reach the required output levels, although the feasibility of these devices is still being debated. Altug et al. ${ }^{1}$ have experimentally investigated coupled PhC cavities and found indications of lasing with modulation speeds exceeding $100 \mathrm{GHz}$, but their results have later been questioned. ${ }^{5}$ In this paper, we extend the tightbinding laser rate equation model ${ }^{6}$ to the nanocavity regime by including Purcell enhancement of the spontaneous emission and by investigating the coupling strength of $\mathrm{PhC}$ cavities through finite-difference time-domain (FDTD) simulations. We present simulations of two coupled PhC lasers and show that regimes where the lasers phase-lock exists, but that they are sensitive to detuning effects such as those arising from fabrication imperfections in the $\mathrm{PhC}$.

The dynamics of conventional quantum well diode lasers is well described by the laser rate equations (LREs) ${ }^{7}$

$$
\begin{gathered}
\dot{N}=J-R_{\mathrm{nr}}-R_{\mathrm{sp}}-G S, \\
\dot{S}=\Gamma G S+\Gamma \beta R_{\mathrm{sp}}-\frac{S}{\tau_{\mathrm{p}}}, \\
\dot{\varphi}=\frac{1}{2} \Gamma \alpha\left(G-G_{\mathrm{th}}\right),
\end{gathered}
$$

where $N$ and $S$ are carrier and photon densities, $J$ is the injection current density, $R_{\mathrm{nr}}=A N+C N^{3}$ is the non-radiative rate (surface and Auger recombination, respectively), $R_{\mathrm{sp}}=B N^{2}$ is the spontaneous emission process, $G$ is the gain rate, $G_{\mathrm{th}}$ is the threshold gain, $\Gamma=V / V_{\mathrm{m}}$ is the confinement factor given as the ratio of the active volume to the mode volume, $\tau_{\mathrm{p}}$ is the photon life-time, $\alpha$ is the linewidth enhancement factor, and $\beta$ is the fraction of spontaneous emission emitted into the relevant laser mode. We approximate the gain by $G=v_{\mathrm{g}}$ $G_{0} \ln \left\{\left(N+N_{\mathrm{s}}\right) /\left(N_{\mathrm{tr}}+N_{\mathrm{s}}\right)\right\}$ which is appropriate for quantum well devices, where $v_{\mathrm{g}}$ is the group velocity, $G_{0}$ is the mate-

\footnotetext{
${ }^{\text {a) }}$ Author to whom correspondence should be addressed. Electronic mail: suhr@fotonik.dtu.dk.
}

rial gain, $N_{\text {tr }}$ is the transparency density, and $N_{\mathrm{s}}$ is a small parameter that keeps the gain finite at low carrier densities. ${ }^{7}$ In micro-cavities, the spontaneous emission into the cavity mode is enhanced by the Purcell factor $F=6 Q /\left(\pi^{2} V_{\mathrm{n}}\right)$, where $Q=\omega_{\mathrm{c}} \tau_{\mathrm{p}}$ is the quality factor of the cavity, $V_{\mathrm{n}}=V_{\mathrm{m}} /\left(\lambda_{\mathrm{c}}\right)$ $\left.\left(2 n_{\mathrm{c}}\right)\right)^{3}$ is the normalized mode volume, and $\omega_{\mathrm{c}}$ and $\lambda_{\mathrm{c}}$ are, respectively, the resonance (angular) frequency and wavelength of the cavity. ${ }^{8}$ In this case, the total spontaneous emission term in Eq. (1) is replaced by $R_{\mathrm{sp}}=B N^{2}(1+\beta(F-1))$, and the spontaneous emission going into the cavity $\beta B N^{2}$ is replaced by $\beta F B N^{2} .5$ We notice that the actual Purcell enhancement of the emission depends not only on the cavity but also on the emitter linewidth, see Refs. 9 and 10. For a weakly coupled two-cavity system (A and B), the coupling can be described by

$$
\dot{E}_{n}=i \omega_{n} E_{n}+\frac{1}{2} i \kappa \omega E_{m}, \quad n \neq m \in\{A, B\},
$$

where $E_{n}$ is the complex electric field in cavity $n$ with resonance frequency $\omega_{n}, \kappa$ is the coupling constant, and $\omega=\left(\omega_{n}+\omega_{m}\right) / 2$. We now split Eq. (4) into equations for the photon density, $S_{n}$, and phase, $\varphi_{n}$, using $E_{n}=\sqrt{S_{n}} \exp$ $\left(i \omega_{n} t+i \varphi_{n}\right)$, so that $S_{n}=\left|E_{n}\right|^{2}$ and $\varphi_{n}=-\omega_{n} t$ $-i \ln \left(E_{n} / E_{n}^{*}\right) / 2$. Inserting Eq. (4) in $\dot{S}_{n}$ and $\dot{\varphi}_{n}$, we arrive at

$$
\begin{aligned}
& \dot{S}_{n}=\kappa \omega \sqrt{S_{n} S_{m}} \sin \left(\delta \omega_{n m} t+\delta \varphi_{n m}\right), \\
& \dot{\varphi}_{n}=\frac{1}{2} \kappa \omega \sqrt{\frac{S_{m}}{S_{n}}} \cos \left(\delta \omega_{n m} t+\delta \varphi_{n m}\right),
\end{aligned}
$$

where $\delta \omega_{n m}=\omega_{n}-\omega_{m}$ and $\delta \varphi_{n m}=\varphi_{n}-\varphi_{m}$. The modified laser rate equations for each of the two coupled cavities are then given by Eqs. (1)-(3) with the coupling terms in Eqs. (5) and (6). For coupled waveguides, the coupling strength $\kappa$ is usually defined from an overlap integral between neighboring modes. ${ }^{7,11}$ The dissipative nature of cavity modes, however, renders this approach highly ambiguous since the modes necessarily diverge exponentially at long distances, ${ }^{12}$ calling for a non-Hermitian reformulation of the coupling equations. This is beyond the scope of the present work, so, we take a more direct approach based on time-domain calculations. In a system of two resonant and passive cavities 


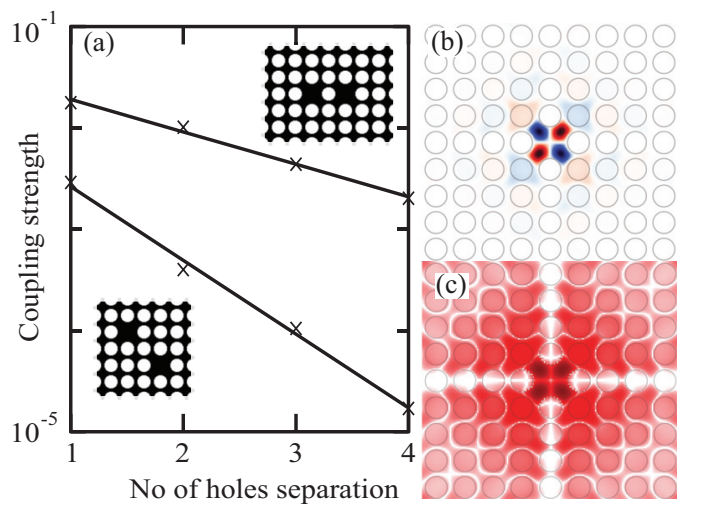

FIG. 1. (Color online) (a) Calculated coupling strength for two cavities with varying intercavity distance in both the $\Gamma-\mathrm{X}$ and $\Gamma-\mathrm{M}$ direction. The solid lines are exponential fits. (b) Mode profile for the quadrupole mode. (c) Absolute square of the quadrupole mode.

described by Eq. (5), exciting a mode in only one cavity leads to an oscillating field amplitude as energy is exchanged between the cavities. It can be shown that the field amplitude oscillates at a frequency given by $\omega_{\mathrm{TB}}=\kappa \omega / 2$. In this way, the coupling constant can be derived from the time evolution of the field amplitude, which is easily obtained from FDTD simulations. Figure 1(a) displays example calculations of the coupling strengths for varied intercavity spacing in both the $\Gamma-\mathrm{X}$ and $\Gamma-\mathrm{M}$ direction for a quadrupole mode (see Figures 1 (b) and 1(c)). We note an exponential decrease in the coupling strength for both directions, but the decrease is faster for the $\Gamma$-M direction corresponding to the cavities being $\sqrt{2}$ further apart. The data in Figure 1(a) indicate a maximum coupling strength of approximately $10^{-2}$ for photonic crystals, and while there is, in principle, no lower limit for the coupling strength, the coupling terms in real systems must be large enough to overcome the various noise terms.

The differential equations were solved numerically with the initial conditions set to $N_{A}=N_{B}=N_{\text {tr }}$ and $S_{A}=S_{B}=0.1 N_{\text {tr. }}$. It is clear from Eqs. (5) and (6) that a phase difference of any integer multiple of $\pi$ will lead to in- and out-of-phase solutions that evolve independently of each other. In practice, random noise will perturb these solutions and introduce a coupling, and we, therefore, choose an initial phase difference $\delta \varphi=\pi / 2$. In all the simulations, the parameters given in the caption to Figure 3 were used unless otherwise specified. Notice that we here consider a class B laser which, with the given parameters, have a carrier lifetime at transparency of $0.5 \mathrm{~ns}$. Solving the modified equations leads to either constant and phase-locked solutions or oscillating solutions depending on the input parameters and the coupling constant. Fig. 2 shows Poincaré maps ${ }^{13}$ of the solutions for two coupled cavities as function of $\kappa$. Looking first at a zero detuning case (Fig. 2, top), we see that for negligible coupling $\left(\kappa<4 \times 10^{-6}\right)$, the cavities reach constant steady-state solutions and are phase-locked with a constant phase difference (phase-locking regime I). For $\kappa \approx 4 \times 10^{-6}$, the steady-state solutions begin to oscillate harmonically, which is seen as a bifurcation in the Poincaré map. Further increasing the coupling strength leads to more and more bifurcations giving very complicated oscillatory behavior (see insets in Fig. 2). At large coupling strengths $\left(\kappa>2 \times 10^{-4}\right)$, the complicated oscillations suddenly collapse, and a new phase-locking regime

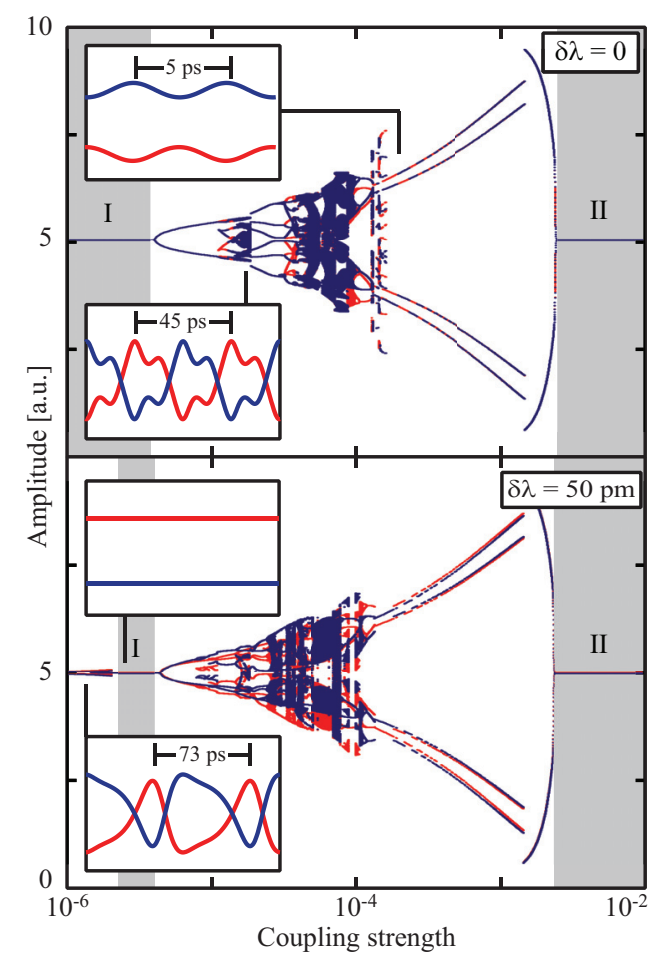

FIG. 2. (Color online) Poincaré map of the steady-state solutions for two coupled cavities for detunings of $0 \mathrm{pm}$ (top) and $50 \mathrm{pm}$ (bottom). The insets show examples of the time evolution of the photon densities at different coupling constants. The colors indicate the amplitudes in cavities $\mathrm{A}$ and $\mathrm{B}$, and the shaded areas mark the phase-locking regimes I and II.

appears (phase-locking regime II). Due to imperfections in the fabrication processes, the laser cavities will in general differ from each other with variations in the characteristic parameters, including Q-factor, mode volume, and resonance frequency. In the bottom part of Fig. 2, we show the Poincaré map for a detuning of $50 \mathrm{pm}$. In general, the Poincaré map is relatively unchanged except that phase-locking regime I becomes limited from below, where the coupling is dominated by detuning effects. The introduction of detuning is seen to reduce the extent of both phase locking regimes, with regime I being affected at even minute values of the detuning. In both regimes I and II, the steady-state photon densities split due to the coupling.

Figure 3(a) shows the dependence of the phase-locking regimes on the coupling strength and detuning (relative to the cavity linewidth), showing that the extent of regimes I and II is strongly affected by detuning, with regime I disappearing for detuning values larger than a critical value that increases with coupling strength. For realistic detunings imposed by fabrication disorder (a few nanometers), the lower phase-locking bound is already above $10^{-3}$ and is therefore an important factor to take into account.

Figures 3(b)-3(d) show the extent of the phase-locking regimes as the $\alpha, \beta$, and Purcell factors are varied, with solid (dashed) lines corresponding to $\delta \omega=50 \mathrm{pm}(\delta \omega=0)$. Figures 3(b) and 3(c) show that surprisingly high values of $\beta$ and $F$ are needed to achieve phase-locking, and that even for zero detuning phase-locking, regime II only opens at quite high values of $\beta$ and $F$. On the other hand, Figure 3(d) shows that phase-locking is possible in a much broader range for lower values of the linewidth enhancement factor in agreement with 


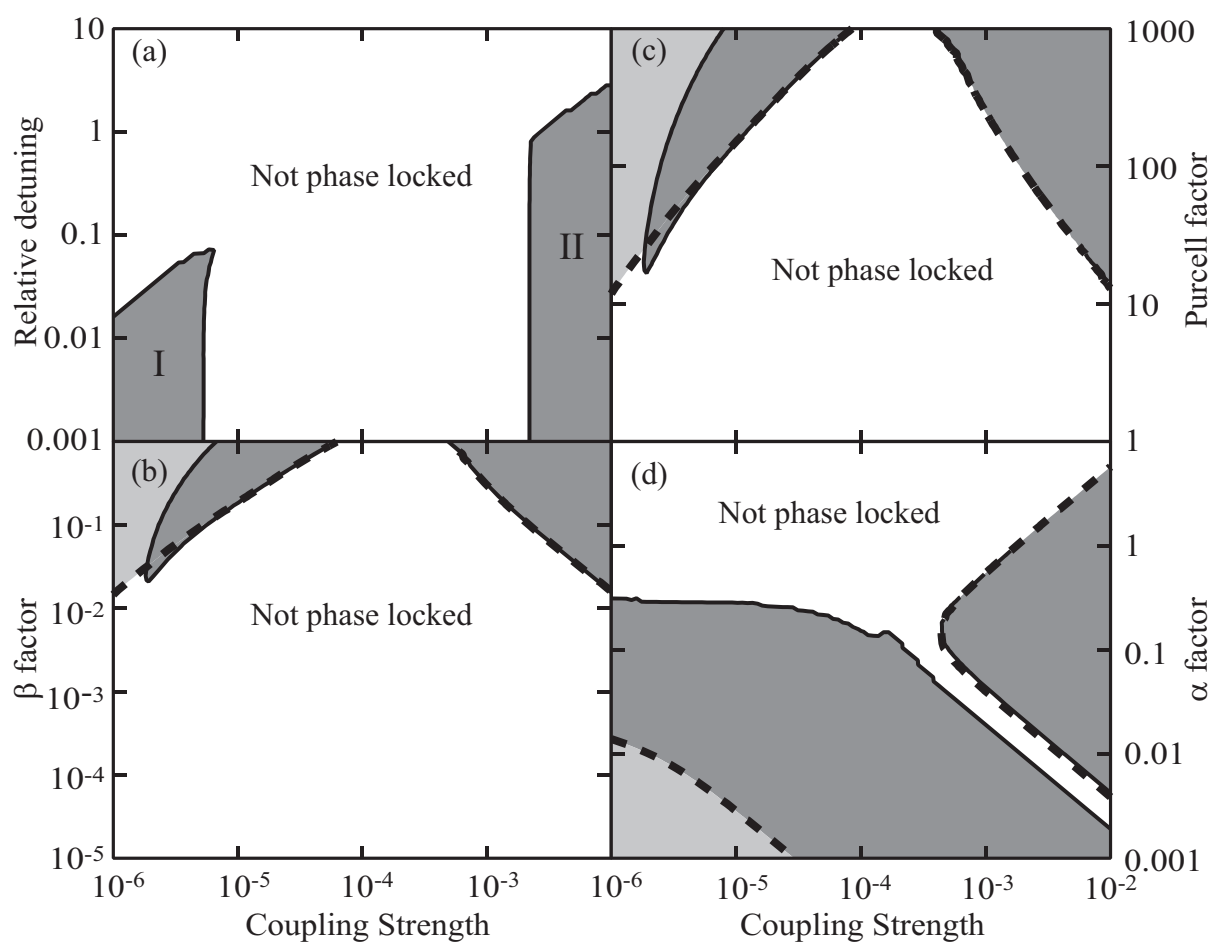

FIG. 3. (a) The extend of the phase-locking regimes is shown in the plane of coupling strength and detuning (relative to the cavity linewidth). The shaded areas are phaselocked. (b)-(d) The extend of the phase-locking regimes for zero detuning (dashed line) and for $\delta \lambda=50 \mathrm{pm}$ (solid line) in the plane of coupling strength and (b) the $\beta$ factor, (c) the Purcell factor and (d) the linewidth enhancement factor. The fixed parameters are (Ref. 7): $n_{\mathrm{c}}=3.5, \Gamma=0.1, \beta=0.1, \alpha=4$, $\lambda_{\mathrm{c}}=937 \mathrm{~nm}, \mathrm{Q}=1250, V_{\mathrm{n}}=15, \mathrm{~F}=50$, $G_{0}=0.18 \mu \mathrm{m}^{-1}, \quad N_{\mathrm{tr}}=1.8 \times 10^{6} \mu \mathrm{m}^{-3}, \quad$ A $=10^{-3} \mathrm{ps}^{-1}, \mathrm{~B}=1.8 \times 10^{-4} N_{\mathrm{tr}}^{-1} \mathrm{ps}^{-1}, \quad \mathrm{C}$ $=3.2 \times 10^{-5} N_{\mathrm{tr}}^{-2} \mathrm{ps}^{-1}, \mathrm{~N}_{\mathrm{s}}=1.8 \mu \mathrm{m}^{-3}$.
Ref. 6. Quantum dot devices may be advantageous in this respect since they feature smaller values of $\alpha$, but the dynamics also differs and additional investigations are required.

In conclusion, we have theoretically analyzed coupled nanocavity lasers and identified phase-locking regimes for two coupled cavities as well as their dependence on various design and material parameters. Based on finite difference time domain calculations of two cavities in a rectangular photonic crystal, we extracted the coupling constant and found it to be less than $\kappa \approx 10^{-2}$. Together with the parameter analysis, this suggests that phase-locked operation of arrayed nanocavities is difficult to achieve in quantum well systems due to the relatively large linewidth enhancement factors and low Purcell factors for these structures. It is possible that quantum dot systems are more suitable than quantum wells due to their low linewidth enhancement factor and the possibility of realizing high Purcell enhancement.

The authors thank Villum Fonden for financial support via the NATEC (Nanophotonics for TErabit Communications) center.
${ }^{1}$ H. Altug, D. Englund, and J. Vuckovic, Nat. Phys. 2, 484 (2006).

${ }^{2}$ R. Hostein, R. Braive, L. L. Gratiet, A. Talneau, G. Beaudoin, I. Robert-Philip, I. Sagnes, and A. Beveratos, Opt. Lett. 35, 1154 (2010).

${ }^{3}$ M. Nomura, Y. Ota, N. Kumagai, S. Iwamoto, and Y. Arakawa, Appl. Phys. Lett. 97, 191108 (2010).

${ }^{4}$ M. Nomura, N. Kumagai, S. Iwamoto, Y. Ota, and Y. Arakawa, Opt. Express 17, 15975 (2009).

${ }^{5}$ E. K. Lau, A. Lakhani, R. S. Tucker, and M. C. Wu, Opt. Express 17, 7790 (2009).

${ }^{6}$ H. G. Winful and S. S. Wang, Appl. Phys. Lett. 53, 1894 (1988).

${ }^{7}$ L. A. Coldren and S. W. Corzine, in Diode Lasers and Photonic Integrated Circuits, edited by K. Chang (John Wiley \& Sons, Inc., New York, 1995).

${ }^{8}$ J. M. Gerard and P. Michler, Single Quantum Dots: Fundamentals, Applications and New Concepts (Springer, New York, 2004), Chap. VII, pp. 269-309.

${ }^{9}$ T. Suhr, N. Gregersen, K. Yvind, and J. Mørk, Opt. Express 18, 11230 (2010).

${ }^{10}$ T. Suhr, N. Gregersen, M. Lorke, and J. Mørk, Appl. Phys. Lett. 98, 211109 (2011).

${ }^{11}$ A. Yariv, Optical Electronics (Holt McDougal, New York, 1985).

${ }^{12}$ K. M. Lee, P. T. Leung, and K. M. Pang, J. Opt. Soc. Am. B 16, 1409 (1999).

${ }^{13}$ G. B. Arfken and H. J. Weber, in Mathematical Methods for Physicists, 5th ed., edited by A. Dooley (Academic, San Diego, California, 2001). 